\title{
Summary Talk: Field Theory
}

\author{
J. Barcelos-Neto \\ Instituto de Física \\ Universidade Federal do Rio de Janeiro, \\ Rio de Janeiro, RJ, 21945-970, Brazil
}

Received on 9 March, 2001

\begin{abstract}
We present a summary of the contributions on Field Theory at the XXII Brazilian National Meeting on Particles and Fields.
\end{abstract}

In the meeting of this year, there were a total of 165 contributions in the form of panel and oral sections, besides two plenary and three parallel talks. As occurred in the previous meetings, these talks refer to recent and/or significative subjects in the Field Theory. At this year, one talk was on the Anti-de Sitter (AdS)Conformal Field Theory (CFT) correspondence, two on nonconcommutative theories, one on application of field theory in condensed matter and one on derivative expansions and finite temperature. I find also important to mention that there were 14 contributions from the Cosmology and Gravitation sector which also concerns to field theory. The quantum and semiclassical aspects of gravitation as well as quantum fields in classical and curved background are subjects whose interest has been increasing year after year.

It might be tedious and uninteresting to make an analysis of each contribution (or group of them) isolated from the general view of where this work is in the context of the Field Theory as a whole. The main reason is that one of the purposes of this talk is to let people of other areas know what has been done in Field Theory.

In my opinion, another important point would be to situate the works within the development of the corresponding research groups in Brazil in order to have an idea of what has been done in each group during the last years. Concerning this last part, we refer to the careful analysis of Prof. Marcelo Gomes in the summary talk of the last meeting. I am going to concentrate on the first part.

We may say that the success of the Field Theory starts from the quantization of the electromagnetic and fermion fields, describing the electromagnetic interaction (QED), where there is a fantastic agreement with experiments. QED is a gauge theory whose symmetry group is the $U(1)$. In the year 1954, Yang and Mills proposed an extension for this gauge theory in order to include non-Abelian fields. This was achieved by considering more general groups $S U(N)$, where the number of gauge fields is $N^{2}-1$. Even though considered a very nice theoretical idea, the first successful application of the Yang-Mills fields just occurred almost fifteen years later (1968), in the consistent description of the weak interaction in a unified theory also involving QED. The corresponding symmetry group is the $S U(2)_{L} \otimes U(1)_{Y}$ (where "L" means "left doublets" and "Y" refers to the hypercharge) and its development was due to Glashow, Salam and Weinberg. It is opportune to mention that the experimental observation of weak gauge fields took fifteen years more, after the construction of powerful accelerators.

Later on, the strong interaction was also formulated as a Yang-Mills theory, whose symmetry group is the $S U(3)$ and the basic ingredients are not protons and neutrons, as in the old Nuclear Physics, but quarks and gluons. Nowadays, the gauge theory whose symmetry group is $S U(3) \otimes S U(2)_{L} \otimes U(1)_{Y}$, also known as standard model, correctly explains all the events we know involving weak, electromagnetic and strong interactions. The standard model and problems related with confinement, vacuum QCD etc. are always an interesting area of research in quantum field theory $[1,2,3,4]^{1}$. We also mention that problems related to the Casimir Effect have increased of interest in recent years $[5,6,7,8,9,10,11,12]$.

The path integral formalism in Field Theory has a very high resemblance with the partition function in Statistical Physics. The intersection of these initially distinct subjects leads to a fruitful line of research because the knowledge of each one could be used into the other. The so called Quantum Field Theory at Finite Temperature, or Thermal Field Theory, has always been an interesting research subject

\footnotetext{
${ }^{1}$ These references concern the papers of the meeting. There are works which may appear in more than one place. I apologize for references put in a wrong way.
} 
$[13,14,15,16,17,18,19,20,21,22,23,24]$. In the meeting of this year, it was devoted a parallel talk on this subject, involving the Derivative expansion technique and Chern-Simons theories [25].

Of course, quantum field theories were initially formulated for the spacetime dimension where we live, $D=4$. However, a great deal of interest emerged for quantum fields at lower dimensions, $D=3$ and $D=2$ (even at $D=1$ ). These research lines were initially considered as just a theoretical laboratory for field theories at $D=4$. There are many interesting research areas at this part, involving Chern-Simons theories [26, 27, 28, 29, 30, 31, 32, 33, 34, 35, 36, 37, 38, 39], Anyons and Fractons [18, 40], Nonlinear Sigma-model [41, 42, 43, 44], Schwinger and Chiral-Schwinger models $[45,46]$ etc. Nowadays, field theories at spacetime dimensions lower than $D=4$ still have a plenty of interest by their own rights $[47,48,49,50,51,52,53,54,55,56$, $57,58,59,60,61,62,63,64,65,66,67,68,69,70,71]$ and not only as a theoretical laboratory for $D=4$. Further, they have also connection with the real world, in a research area involving condensed matter with many interesting applications $[72,28,73,74,75,76,77,78,79]$. It was also devoted a parallel talk on this subject [80].

After the success of the field quantization method in the weak, electromagnetic and strong interactions, the most natural step would be to use the same quantization rules into the Einstein theory of gravity. This did not work! The main reason is that quantum field theories deal with many infinite quantities which are either simple discarded, as the vacuum energy, or are intelligent circumvented (the renormalization program). Both these procedures cannot be applied to the gravity theory. First because sources of energy cannot be simple discarded in presence of gravity and second because the renormalization program simply does not work.

A first attempt to circumvent this problem was to follow the same idea before the advent of QED, that is, just quantum matter fields were quantized and interacting with a classical electromagnetic background. The corresponding research area of quantum matter and quantum gauge fields propagating in a classical gravitational background, and the problems related with geometrization, lead to a very interesting developments and constitute a very fruitful research area $[81,82,83,84,85,86,87,88,89,90,91]$.

The current idea on this subject is that there must exist some more general formulation for the gravitational theory where the quantization procedures can be applied. The first consistent attempt was based on the supersymmetry, that is an extension of the Lorentz symmetry where the spacetime also has fermionic degrees of freedom. The corresponding supersymmetric theory of gravity, called supergravity, was then formulated and it contains the Einstein gravitational theory as a particular case. Both supersymmetry and supergravity always deal with interesting research problems to be solved
[92, 93, 94, 95, 96, 97, 98, 30, 90, 99, 100, 101, 102].

However, the problems related with the infinities of quantum gravity were not completely solved with supergravity. An important step was done based on the idea that fields could not depend on points (considered to be a mathematical idealization), but on extended objects. Strings are the simplest extended objects. However, a field theory where fields are functions (or functionals) of strings is very difficult to be handled. What remained is the string idea itself, where elementary particles are not points, but vibrational states of strings. More general extended objects, the branes, were also considered, but it is opportune to say that they are not exactly the same of the modern branes. These are related to boundary surfaces described by strings. Anyway, string and branes (with their supersymmetric version) are a very fruitful research area $[103,104,105$, 106, 107, 87, 108, 109, 110, 111, 112, 113, 114]. There was a plenary talk involving string theory (and noncommutative fields) [115].

The mathematical structures of strings and branes are much more involved and their quantization led to a great development in the quantization methods and in the study of anomalies $[116,117,118,119,120,121$, $122,123,124,125,126,127,128,129,130,131]$. The treatment of constrained systems (Dirac, symplectic, Senjanovic etc. $[132,133,134,135,136,112,38,137]$ were nicely improved, based in the BRST symmetry. The works of Batalin, Fradkin, Vilkovisky and Tyutin are very relevant in the quantizations methods known in literature as BFV (Hamiltonian), BV (Lagrangian), and BFT (Hamiltonian embedding) [41, 138, 139, 140]. Mathematical structures and their algebras, as well as problems related to Lax pairs, KP hierarchy, integrable models acquired much interest $[141,142,143,144,145$, $34,146,147,148]$.

Another aspect of extended objects is that they are consistently quantized just in the spacetime dimension $D=10$. This might means that there exists some compactification procedure which leads to our spacetime dimension [28, 149]. There is another interesting aspect in (super)string theories. At first, it appeared to exist five independent theories for them. But now we know that they are connected by duality, together with supergravity at $D=11$ (we are going to explain below why an extra dimension appears). It is important to emphasize that supergravity is again a very interesting research area. The consequence of the duality among string and supergravity theory is that they must be different manifestation of a some fundamental theory, called $M$-theory in literature, that should be formulated at $D=11$. In this line of research, problems related to duality $[150,151,152,153,39,154]$ and topology $[155,98,156,157,158,154,159,160,161,162]$ have a great deal of interest.

After a brilliant work due to J. Maldacena, published in 1998, the old idea (and dream) of a total uni- 
fication and to have a unique theory able to describe everything is again in evidence. As it was said, there were problems to include gravitation in the family of quantum gauge theories. On the other hand, after the advent of strings, the problem has changed in a reversed way. String theories naturally contain gravity, but the difficulty was to include Maxwell and Yang-Mills theories into this formalism. Maldacena conjectured that there exists a duality between (super)string theories or supergravity in $A d S_{D+1}$-spaces and conformal gauge theories living in the boundary at $D$-dimensions (that is why the previous critical dimension $D=10$ of superstrings has changed do $D=11$ ). It is true that a unified theory of everything might be still far, but the duality among all the string theories and supergravity at $D=11$ and the work of Maldacena have certainly shed new light on this old dream. This line of research, including the recent works of Lisa Randall and Raman Sundrum and the possibility of noncommutative fields, is the most recent subjects in quantum field theory $[163,164,165,166,167,168]$. There was a parallel talk on $A d S / C F T$ correspondence [169] and a plenary talk on noncommutative fields $[115,170]$. It might be opportune to say that it was also planned a plenary talk on $A d S / C F T$ with Maldacena himself, that had confirmed but declined few weeks before the meeting by virtue of personal problems.

\section{References}

[1] R.C. Arcuri and M. Novello, construindo bosons de Higgs com férmions auto-interativos.

[2] A.E. de Bernardini, Desenvolvimento de modelos unifcados com aplicação no estudo de simetrias "left-right".

[3] M.A.C. Torres, Estudo de confinamento em cordas não críticas.

[4] P.P. Srivastava and S.J. Brodsky, Standard model of electro-weak interactions in light-cone gauge: a renormalizable, unitary, and ghost-free theory.

[5] D.T. Alves, C. Farina, P.A. Maia Neto, and A.C. Tort, Dynamical Casimir effect with Neumann condition at finite temperature.

[6] J.C. da Silva, A. Matos Neto, A.E. Santana, and F.C. Khanna, Generalized Bogoliubov transformation in thermofield dynamics and the Casimir effect.

[7] R.B. Rodrigues and N.F. Svaiter, Vacuum stress tensor of a scalar field in a retangular waveguide.

[8] J.A. Nogueira, On the effects of homogeneous Dirichlet's boundary conditions in the spontaneous symmetry breaking of a real scalar field.

[9] R.M. Cavalcanti, Força de Casimir entre placas semitransparentes.

[10] V.S. Mendes and A.E. Gonçalves, A fórmula de CardyVerlinde revisitada.

[11] M.V. Cougo-Pinto, C. Farina, and J.F.M. Mendes, $C a$ simir effect in kappa deformed electrodynamics.
[12] M.V. Cougo-Pinto, C. Farina, A.C. Tort, F.A. Farias, and M.S. Ribeiro, Casimir energy of Dirac field in external magnetic field by the method of generalized zeta function.

[13] C.A. Aragão de Carvalho and A.J. Silva, Alternative dimensional reduction.

[14] F.C. Cabral and E.R. Bezerra de Mello, Polarização de vácuo por campo fermiônico sem massa no espaçotempo de um monopolo global a temperatura não nula.

[15] R.O. Ramos, Testing quantum field theory methods in experiments of Bose-Einstein condensation.

[16] M.M. Leite and L.C. de Albuquerque, Pontos de Lifshitz anisotrópicos em $O\left(\epsilon_{L}^{2}\right)$.

[17] M.M. Ferreira Jr., O.M. Del Cima, and J.A. HelayëlNeto, High- $T_{c}$ cuprate suprconductors and a planar QED model.

[18] P.F. Borges, H. Boschi Filho, and M. Hott, Coeficientes do virial a partir de açôes efetivas para a eletrodinâmica quântica em $(2+1)$-dimensões a temperatura e denside finitas.

[19] F.R. Machado and F.T.C. Brandt, Perturbações gravitacionais em um meio térmico.

[20] M. Moutinho, W.A. Moura-Neto, S.M. de Souza, M.T. Thomaz, Modelo de Thirring a altas temperaturas.

[21] H.C.G. Caldas, The damping rate of a fermion within the linear sigma model at finite temperature.

[22] W.A. Moura-Melo, S.M. de Souza, O. Rojas, and M.T. Thomaz, High temperature expansion of the spin-1 Heisemberg XXZ chain.

[23] M.H.G. Pacheco and R.R. Landim, O oscilador de Dirac num banho térmico.

[24] H. Queiroz and J.L. Tamazelli Operador espalhamento $\hat{S}$ no espaço de Fock para QED a temperatura finita.

[25] F. Brandt, Expansão derivativa em ordens superiores $e$ a ação efetiva para a teoria de Chern-Simons abeliana e não-abeliana.

[26] H. Belich, M.M. Ferreira Jr., O.M. Del Cima, and J.A. Helayël-Neto, Electron-electron attactive interaction in Maxwell-Chern-Simons $Q E D_{3}$ at zero temperature.

[27] J.A. Helayël-Neto and W.A. Moura-Melo, Some consequences of the quantum violation of the Huyghens principle on the behavior of radiation in Maxwell and Maxwell-Chern-Simons models in three dimensions.

[28] J. Barcelos-Neto and E.C. Marino, Uma nova seqüência para termos topológicos em qualquer dimensão do espaço-tempo.

[29] L.E. Oxman and S.P. Sorella, Vortex correlation functions in nonlocal Maxwell-Chern-Simons models.

[30] E.C.A. Nogueira, J.L. Boldo, O. Piguet, and A.M. Delpupo, Supersimetria vetorial em teoria de ChernSimons e gravitação em 3-dimensões.

[31] J.L. Boldo, L.M. de Moraes, and J.A. Helayël-Neto, Remarks on propagating torsion.

[32] A.M. Delpupo, J.L. Boldo, and O. Piguet, ChernSimons versus gravitação em 3 e 5 dimensões. 
[33] J.L.A. Fernandes and D.H.T. Franco, Asymptotic scale invariance of a non-Abelian Chern-Simons matter model.

[34] J.A. Helayël-Neto and R.C. Paschoal, Quantum mechanical aspects of charged particles in Maxwell-ChernSimons electrodynamics.

[35] A.P.B. Scarpelli and J.A. Helayël-Neto, Vortex lines and Dirac magnetic monopoles for the Abelian Higgs model with Lorentz CPT violation.

[36] J.A. Helayël-Neto, M.A. Santos, and P.I. Trajtenberg, Probing the symplectic projector method of quantization in planar gauge models.

[37] P.T.B. Crispim, R.R. Landim, and C.A.S. de Almeida, Nonminimal Chern-Simons-Higgs model: broken-symmetry phase.

[38] K.C. Mendes, R.R. Landim, and C.A.S. de Almeida, Dirac quantization of a nonminimal Chern-Simons gauged $O(3)$ sigma-model.

[39] D. Bazeia, A. Ilha, J.R.S. Nascimento, R.F. Ribeiro, and C. Wotzasek, On the equivalence of the BornInfeld-Chern-Simons model coupled to dynamical U(1) charged matter.

[40] W. da Cruz, Fractons and fractal-deformed Heisemberg algebras.

[41] J. Ananias Neto, A. Deriglazov, W. Oliveira, and G. de Oliveira Neto, Equivalence between Dirac first-class constraints and reduced phase space quantization of the $O(N)$ non-linear sigma model.

[42] M.S. Cunha, Vórtices assimétricos no modelo $\sigma-O(3)$ não minimamente acoplado.

[43] M.S. Góes-Negrão, J.A. Helayël-Neto, and M.R. Negrão, Coupling of a nonlinear $\sigma$-model to a less constrained non-Abelian (2,0)-super Yang-Mills.

[44] M.S. Góes-Negrão, J.A. Helayël-Neto, and M.R. Negrão, Spin 2 ressonances from a nonlinear $\sigma$-model.

[45] E.M.C. Abreu and A.S. Dutra, Remarks on the physical mechanism behind the soldering formalism.

[46] E.M.C. Abreu and A.S. Dutra, Bosonized QED with mixed quartic interaction terms for Dirac ferminons.

[47] F. Braghin, Expanding configurations of the $\lambda \phi^{4}$ model.

[48] V.I. Fernánde C.A. Iucci, and C.M. Naón, Nonlocal Thirring model including backward and Umklapp scattering processes.

[49] J.A.E. Carrillo and A. Maia Jr., Linear and nonlinear fluctuations in $\lambda \phi^{4}$ theory in a finite domain: Hirota's method approach

[50] A.P.C. Malbouisson and J.M.C. Malbouisson Boundary dependence of the coupling constant in the vector $N$-component $\left(\lambda \phi^{4}\right)_{D}$ model.

[51] L.V. Belvedere, R.L.P.G. do Amaral, and A.F. de Queiroz, The fermion-boson mapping applied to Lagrangian models for charge-density-waves in quasi-one-dimensional systems.

[52] L.P. Collato, A.L.A. Penna, W.C. dos Santos, C.M.M Polito, (1,1) matrix world from a $(2 n+1,2 n+1)$ ordinary one vector case.
[53] E.M.C. Abreu, M. Hott, J.A. Helayël-Neto, and W.A. Moura-Neto, On Dirac-like monopoles in three dimensions and some possible invluences on the dynamics of charged particles.

[54] D. Bazeia, A.S. Inácio, and L. Laercio, Some new domain wall solutions of the BPS type.

[55] S.P. Gavrilov, D.M. Gitman, and A.A. Smirnov, Green functions of the relativistic wave equations for constant homogeneous external field in the presence of Aharonov-Bohm potential, and their applications.

[56] A.N. Vaidya and L.E.S. Souza Algebraic calculation of the $S$-matrix for the Dirac-Coulomb problem in $2+1 \mathrm{di}$ mensions.

[57] A.N. Vaidya and L.E.S. Souza Algebraic calculation of the S-matrix for a generalized Dirac-Coulomb problem dimensions.

[58] A.N. Vaidya and L.E.S. Souza Green function for the Dirac-Coulomb problem in $2+1$ dimensions.

[59] A.W. Smith, J.A. Helayël-Neto, and R.S. Simões, $E x-$ act classical solutions to $(1+1)-D$ wave equations with arbitrary higher derivatives.

[60] J.A. Helayël-Neto and P. Brockill, Causal propagation of spin-2 torsion in 3D-gravity.

[61] A.L.M.A. Nogueira, J.A. Helayël-Neto, and M.E.X. Guimarães, Coupled $U(1) \times U(1)$ vortices.

[62] W.G. Ney and J.A. Nogueira, Geração de massa em uma região espacial finita de dimensão $(1+1)$.

[63] M.S. Góes-Negrão, J.A. Helayël-Neto, and M.R. Negrão, Gravitation in $D=1+2$ and $C P^{N} \sigma$-model.

[64] M.M. Ferreira Jr., R.B. Ribeiro, O.M. Del Cima, and J.A. Helayël-Neto, The parity-preserving $U(1)_{A} \times U(1)_{a}$ $Q E D_{3}$ at 1-loop.

[65] M.O. Tahim, R.R. Landim, and C.A.S. de Almeida, Estudo da equação de Dirac em $2+1$ dimensões para um potencial coulombiano com momento de dipolo magnético anômalo.

[66] W.G. Pereira and A.S. de Castro, Explorando a caixa de Dirac

[67] O.M. Del Cima, D.H.T. Franco, O. Piguet, and M. Schweda, No parity anomaly in massless $Q E D_{3}$.

[68] R.S. Silva and J.A. Helayël-Neto, Vector central charges in $D=3$ dimensions.

[69] R.L. Rodrigues, A.F. de Lima, K.A. Ferreira, and A.N. Vaidya, Calogero interaction in the minimum uncertainty coherent states.

[70] G. Flores and N.F. Svaiter, Constructing bidimensional scalar field theory models from zero mode fluctuations.

[71] E.W. Dias and A.L. Mota, Cálculos não perturbativos no modelo $\lambda \phi^{4}$ em $1+1$ dimensões usando o método dos elementos finitos.

[72] C. Farina and A.C. Tort, Dispersive van der Waals forces.

[73] C.A.G. de Almeida, W.H.C. Freire, D. Bazeia, and R.F. Ribeiro, On the existence of internal modes of solitary waves. 
[74] J.A. Helayël-Neto, W. Spalenza, and T.C. Soares, $A$ $Q E D_{3}$ formulation of vortices in boson condensates.

[75] V.B. Bezerra, R. Menezes, and J.R.S. Nascimento, Bosonic condensate near a superconducting cosmic string.

[76] D.T. Alves, F.A.B. Rangel, C. Farina, and A.C. Tort, On the influence of two parallel plates on the atomic levels.

[77] J. Acosta and H. Blas, Generalized sine-gordon/massive thirring models and soliton/particle correspondences.

[78] D.Bazeia e R.F. Ribeiro, Chiral polarons in single walled carbon nanotubes.

[79] G. Flores and N.F. Svaiter, Vacuum fluctuations of a scalar field in a rectangular waveguide.

[80] D. Bazeia, Campos escalares: das paredes de domínio aos nanotubos e fulerenos.

[81] R.D.M. de Paola and N.F. Svaiter, A rotating vacuum and a quantum version of newton's bucket experiment.

[82] S.I. Zlatev, World-line path integral for fermions amplitudes.

[83] R. Casana, J.T. Lunardi, B.M. Pimentel, and R.G. Teixeira, DKP field in Riemann-Cartan space-times.

[84] L. Manssur and R. Portugal, Group-theoretical approach for symbolic tensor manipulation: II. Dummy indices.

[85] A. Accioly and H. Mukai, Potencial efetivo não relativístico para a teoria de gravitação de ordem superior em $D$ dimensões.

[86] J.P.S. da Silva, U. de Freitas, and E.R. Bezerra de Mello, Monopolo magnético gravitacional no espaçotempo do monopolo global.

[87] V.B. Bezerra, L.P. Collato, M.E.X. Guimarães, R.M. Teixeira Filho, Scalar and spinor particles in the spacetime of a domain wall in string theory.

[88] V.B. Bezerra, R.M. Teixeira Filho, G. Grebot, and M.E.X. Guimarães, Vacuum polarization in the spacetime of a scalar-tensor cosmic string.

[89] R. Aldrovandi and A.L. Barbosa, Yang-Mills field as optical media.

[90] D.H.T. Franco and C.M.M. Polito, Some structural aspects of a superfield model in a curved background.

[91] A.E. Santana, M. de Montigny, F.C. Khanna, and E.S. Santos Galilei-covariant Bhabha equations.

[92] M.A.C. Kneipp and P. Brockill BPS string solutions in non-Abelian Yang-Mills theories and confinement.

[93] A.W. Smith and J.A. Helayël-Neto, Auxiliary fields in higher-dimensional supergravities: the rôle of p-forms and fermion condensates.

[94] A. Petrov, Superfield effective action in noncommutative Wess-Zumino model.

[95] C.N. Ferreira, M.B. Maia-Porto, and J.A. Helayël-Neto, Cosmic string configuration in the supersym metric CSKR theory.

[96] C.N. Ferreira, C.F.L. Godinho, and J.A. Helayël-Neto, Gauge field mixing in the supersymmetric cosmic string theory.
[97] H.R. Christiansen, J.A. Helayël-Neto, A.L.M.A. Nogueira, and L.R.U. Mansur, N=2 superalgebra of a nonminimum Maxwell-Chern-Simons-Higgs model: establishing the central extension.

[98] C.P. Constantinidis, O. Piguet, and F. Gieres, Sobre a supersimetria vetorial em teorias topológicas e de gravitação.

[99] M.B. Silka, Modelos integráveis supersimétricos de Wess-Zumino-Witten.

[100] G.S. Dias and J.A. Helayël-Neto, N=2-Susy of a spin$\frac{1}{2}$ particle in an extended external field.

[101] M.A. de Andrade and I.V. Vancea, D=4 Euclidean supergravity: the Dirac eigenvalues and its observables.

[102] R.L. Rodrigues, The susy method for the spectrum of the first Pöschl-Teller potential.

[103] N. Berkovits, O. Chandia, and B.C. Vallilo, quantização covariante da supercorda.

[104] Y. Matsuo, Projection operator and D-brane in string theory.

[105] R. Medina, The low energy effective actions for electromagnetism and gravity that come from the open and closed superstring theories

[106] A. Deriglazov, Separated and irreducible constraints for IIB Green-Schwarz superstring.

[107] E.V. Gorbar, V.P. Gusynin, and V.A. Miransky, Dynamical chiral symmetry breaking on a brane in reduced QED.

[108] G.N. Santos Filho and W.F. Chagas-Filho, Invariância de gauge para partículas e cordas.

[109] D. Nedel and N. Berkovits, Type IIB sigma model and constraints of $4 D N=2$ supergravity.

[110] M.C.B. Abdalla, A.L. Gadelha, and I.V. Vancea, Estudo da entropia para a corda bosônica fechada no formalismo de TFD.

[111] A. Deriglazov, C. Neves, W. Oliveira, and J. Ananias Neto, On the canonical quantization of open strings attached at $D_{p}$-branes in the presence of a constant $B$ field.

[112] D.F.Z. Marchioro, Aplicação do método de Dirac à ação da corda bosônica.

[113] G.F. Hidalgo, Extendon (p-brane) solutions in local $n$-form field theories.

[114] M.C. Leite and W. Siegel, Construção de um formalismo covariante para a supercorda aberta em $4=d$ (big picture).

[115] Y. Matsuo, Tachyon condensation and noncommutative geometry in string theory.

[116] F. Toppan, Anomalous effects in classical dynamical systems.

[117] V.E.R. Lemes, S.P. Sorella, O.S. Ventura, L.C.Q. Vilar, and M.S. Sarandy, An algebraic criterion for the ultraviolet finiteness of quantum field theories. 
[118] M.V. Cougo-Pinto, C. Farina, A.C. Tort, and J.F.M. Mendes, Vacuum polarization in scalar QED under external electric field and deformed periodic boundary condition.

[119] A.S. Dutra and M. Hott, Mapeamento não-local para a teoria $B C S$.

[120] J.L. Boldo and C.A.G. Sasaki, Symmetry aspects of fermions coupled to torsion and electromagnetic fields.

[121] A.T. Suzuki, A.G.M. Schmidt, and E.S. dos Santos, Triângulo de férmions por integração em dimensão negativa.

[122] O.A. Battistel, O.L. Battistel, and G. Dallabona, Meson properties in the $S U(2)$ NJL model.

[123] R. Bentin and A. Suzuki, Equivalência entre a covariantização e a integração em dimensão negativa.

[124] C.A.M. Melo, B.M. Pimentel, and P.J. Pompéia, Schwinger's principle and the B-field formalism for the free electromagnetic field.

[125] A.T. Suzuki and A.G.M. Schmidt, Epsilon-expansion for non-planar double-boxes.

[126] J.T.T. Lunardi, B.M.P. Escobar, J.S.S. Valverde, and L.A.M. Vieira Júnior, Método causal aplicado à eletrodinâmica escalar via equação de Duffin-KemmerPetiau: resultados a um "loop".

[127] R.B. Rodrigues and N.F. Svaiter, Vacuum fluctuations of a scalar field in a rectangular waveguide.

[128] M.J. Salvay and C.M. Naón, On a CFT prediction in the sine-Gordon model.

[129] C.A. Iucci, C.M. Naón, and K. Li, Nonlocal Thirring model with spin flipping interactions.

[130] V.G.C.S. dos Santos and W.G. Pereira, Teoria da perturbação de Rayleigh-Schroedinger versus ambigüidade de ordenamento.

[131] S.V.L. Pinheiro, V.S. Alves, and F.P. Campos, A renormalization group study and the Nambu-JonaLassinio model.

[132] M.A. de Andrade, M.A. dos Santos, and I.V. Vancea, Método dos projetores simpléticos em teorias de campos de gauge.

[133] A.C.R. Mendes, W. Oliveira, C. Neves, and F.I. Takakura, Metafluid dynamics as a gauge theory.

[134] A.C.R. Mendes, J. Ananias Neto, W. Oliveira, C. Neves, and D.C. Rodrigues, Gauging second class systems via symplectic gauge-invariant formalism.

[135] E.M.C. Abreu, D. Dalmazi, and E.A. Silva, The Jacobi identity for Dirac-like brackets.

[136] V.G.C.S. dos Santos and A.S. Dutra, Partícula carregada na presença de um campo mangnético homogêneo sob condiçôes de contorno não triviais.

[137] C.F.L. Godinho, C.N. Ferreira, and M.B.D.S. Maia Porto, Symplectic quantization of the Maxwell and Kalb-Ramond field in the light-cone.

[138] A.R. Fazio, V.E.R. Lemes, M.S. Sarandy, and S.P. Sorella, The diagonal ghost equation Ward identity for Yang-Mills theories in the maximal Abelian gauge.
[139] C.N. Ferreira and C.F.L. Godinho, Triplectic gauge fixing for $N=1$ super Yang-Mills.

[140] J. Ananias Neto, C. Neves, and W. Oliveira, Conversão de sistemas de segunda-classe em primeira baseada em simetrias dos termos de Wess-Zumino.

[141] V.B. Bezerra, E.M.F. Curado, and M.A. Rego-Monteiro, A new class of quantum field theory: perturbative computation.

[142] H.L. Carrion, M. Rojas, and F. Toppan, An N=8 superaffine Malcev algebra and its $N=8$ Sugawara.

[143] H.L. Carrion, M. Rojas, and F. Toppan, Division algebras and extended $N=1,2,4,8 \mathrm{KdVs}$.

[144] E.M.F. Curado and M.A. Rego-Monteiro, Heisemberg type algebras of one-dimensional quantum Hamiltonians.

[145] G. Schieber, Funçôes de partição "twistadas" para teoria de campo conforme com condições de contorno $e$ álgebras de simetrias quânticas.

[146] E.L. da Graça, H.L.C. Salazar, and R.L. Rodrigues, Uma representação da álgebra de virasoro via técnica algébrica de Wigner-Heisemberg em sistema bosônico.

[147] M.A. de Andrade and I.V. Vancea, Spinors in arbitrary dimensions.

[148] H. Queiroz and J.L. Tomazelli, O problema da unitaridade do operador $S$ na teoria de Duffin-Kemmer-Petiau para a s-SED.

[149] L.A. Ferreira and E.E. Leite, Alguns aspectos de teorias de Yang-Mills autoduais reduzidas.

[150] V.O. Rivelles, Dualidade off-shell na teoria de borninfeld.

[151] A. Ilha and C. Wotzasek, Equivalência dual entre os modelos autodual e topologicamente massivo nãoabelianaos.

[152] M.B. Cantcheff, Hodge-type self(antiself)-duality in arbitrary dimension and tensorial rank.

[153] D.M. Medeiros, R.R. Landim, and C.A.S. de Almeida, Dualidade em um modelo topológico não-abeliano em $3 D$.

[154] A. Ilha and C. Wotzasek, Duality equivalence between nonlinear self-dual and topologically massive models.

[155] A.A. Bytsenko and Z.G. Kuznetsova, Field-theoretical description of cohomologies.

[156] R.R. Landim and C.A.S. de Almeida, Geração de massa topológica com termo BF não-abeliano em $D$ dimensões.

[157] E.L. de Graça and R.L. Rodrigues, Defeitos topológicos não BPS associados a dois campos reais acoplados.

[158] A.B. Adib and C.A.S. de Almeida, Kink dynamics in a topological Phi ${ }^{4}$ lattice.

[159] V.G. Lima, V.S. Santos, and R.L. Rodrigues, Configurações topológicas e não topológicas exatamente solúveis.

[160] J.L. Strapasson, V.G. Lima, and R.L. Rodrigues, Configurações topológicas para o potencial de poço duplo invertido. 
[161] V.G. Lima, J.L. Strapasson, and R.L. Rodrigues, Aspectos topológicos e estabilidade de sólitons em $1+1$ dimensões.

[162] O.M. Del Cima, J.M. Grimstrup, and M. Schweda, On the finiteness of a new topological model in $D=3$.

[163] G.S. Lozano, E. Moreno, and F. Schaposnik, Solitons in noncommutative space.

[164] P. Minces and V.O. Rivelles, Noether currents and AdS/CFT correspondence.

[165] A.A. Bytsenko and A.E. Gonçalves, Função de partição e entropia de teorias de campo conforme.

[166] M.A. de Andrade, J.E.F. Milione, and J.L.M. Valle,
O termo de Gauss-Bonnet no modelo de Randall-Sundrum.

[167] M.A. de Andrade, M.A. dos Santos, and I.V. Vancea, Free variables on constraint surface for non-commutative open strings.

[168] R. Amorim and J. Barcelos-Neto, Embedding of the massive noncommutative $U(1)$ theory.

[169] N.R.F. Braga, Quantum fields in anti-de-Sitter spacetime and degrees of freedom in the bulk-boundary correspondence.

[170] M. Gomes, Noncommutative field theories. 